\title{
The Next 700 Network Programming Languages (Invited Talk)
}

\author{
Nate Foster \\ Cornell University \\ jnfoster@cs.cornell.edu
}

\begin{abstract}
Specification and verification of computer networks has become a reality in recent years, with the emergence of domain-specific programming languages and automated reasoning tools. But the design of these frameworks has been largely ad hoc, driven more by the needs of applications and the capabilities of hardware than by any foundational principles. This talk will present NetKAT, a language for programming networks based on a well-studied mathematical foundation: regular languages and finite automata. The talk will describe the design of the language, discuss its semantic underpinnings, and present highlights from ongoing work extending the language with stateful and probabilistic features.
\end{abstract}

\section{CCS CONCEPTS}

- Software and its engineering $\rightarrow$ Formal language definitions; • Networks $\rightarrow$ Programming interfaces;

\section{KEYWORDS}

Domain-specific languages, software-defined networking, formal verification, Kleene algebra with tests, NetKAT.

ACM Reference format:

Nate Foster. 2017. The Next 700 Network Programming Languages (Invited Talk). In Proceedings of 49th Annual ACM SIGACT Symposium on the Theory of Computing, Montreal, Canada, fune 2017 (STOC'17-KEY), 1 pages.

DOI: $10.1145 / 3055399.3081042$

\section{INTRODUCTION}

NetKAT is a domain-specific language for network programming [15]. It offers rich abstractions for specifying the behavior of network devices such as routers and firewalls, and accompanying tools for reasoning formally about network-wide properties such as connectivity and traffic isolation. In this talk, I will describe the design of NetKAT, including primitives for filtering, modifying, and transmitting packets; union and sequential composition operators; and a Kleene star operator that iterates programs. I will also present the semantics of the language, which can be formulated in three different ways: as a denotational semantics based on functions from packet histories to sets of packet histories, as an axiomatic semantics based on a sound and complete deductive system, and as an operational semantics based on finite automata. I will discuss

Permission to make digital or hard copies of all or part of this work for personal or classroom use is granted without fee provided that copies are not made or distributed for profit or commercial advantage and that copies bear this notice and the full citation on the first page. Copyrights for components of this work owned by others than ACM must be honored. Abstracting with credit is permitted. To copy otherwise, or republish to post on servers or to redistribute to lists, requires prior specific permission and/or a fee. Request permissions from permissions@acm.org.

STOC'17-KEY, Montreal, Canada

(C) 2017 ACM. 978-1-4503-4528-6/17/06 ..\$15.00

DOI: $10.1145 / 3055399.3081042$

\begin{tabular}{|c|c|c|}
\hline \multicolumn{3}{|l|}{ Syntax } \\
\hline \multirow[t]{6}{*}{ Predicates } & $a, b::=$ true & Identity \\
\hline & | false & Drop \\
\hline & $f=n$ & Test \\
\hline & $a+b$ & Disjunction \\
\hline & $a \cdot b$ & Conjunction \\
\hline & | $\neg a$ & Negation \\
\hline \multirow[t]{6}{*}{ Programs } & $p, q::=a$ & Filter \\
\hline & $\mid f \leftarrow n$ & Modification \\
\hline & $p+q$ & Union \\
\hline & $p \cdot q$ & Sequencing \\
\hline & $\mid p^{*}$ & Iteration \\
\hline & I dup & Duplication \\
\hline \multicolumn{3}{|l|}{ Semantics } \\
\hline \multicolumn{3}{|c|}{ Denotational $\llbracket p \rrbracket \in \mathrm{H}$} \\
\hline \multicolumn{3}{|c|}{ Axiomatic $\quad \vdash p \equiv q$} \\
\hline \multicolumn{3}{|c|}{ Automata-theoretic $\mathcal{A}(q)$} \\
\hline
\end{tabular}

Figure 1: NetKAT syntax and semantics.

applications of the language to practical compilation and verification problems. Finally, I will present highlights from recent work extending the language with stateful and probabilistic features.

Acknowledgments. NetKAT was developed in collaboration with Carolyn Anderson, Spiros Eliopoulos, Arjun Guha, Jean-Baptiste Jeannin, Dexter Kozen, Konstantios Mamouras, Matthew Milano, Mark Reitblatt, Cole Schlesinger, Alexandra Silva, Steffen Smolka, Laure Thompson, and Dave Walker. This work was supported by NSF (CNS-1111698, CCF-1253165, CNS-1413972, CCF-1422046, ACI1440744, and CCF-1535952), ONR (N00014-15-1-2177), Cisco, Facebook, Fujitsu, and Google.

\section{REFERENCES}

[1] Carolyn Jane Anderson, Nate Foster, Arjun Guha, Jean-Baptiste Jeannin, Dexter Kozen, Cole Schlesinger, and David Walker. 2014. NetKAT: Semantic Foundations for Networks. In ACM SIGPLAN-SIGACT Symposium on Principles of Programming Languages (POPL), San Diego, CA. 113-126.

[2] Nate Foster, Dexter Kozen, Konstantinos Mamouras, Mark Reitblatt, and Alexandra Silva. 2016. Probabilistic NetKAT. In European Symposium on Programming (ESOP), Eindhoven, Netherlands. 282-309.

[3] Nate Foster, Dexter Kozen, Matthew Milano, Alexandra Silva, and Laure Thompson. 2015. A Coalgebraic Decision Procedure for NetKAT. In ACM SIGPLANSIGACT Symposium on Principles of Programming Languages (POPL), Mumbai, India. 343-355.

[4] Steffen Smolka, Spiridon Eliopoulos, Nate Foster, and Arjun Guha. 2015. A Fast Compiler for NetKAT. In ACM SIGPLAN International Conference on Functional Programming (ICFP), Vancouver, BC. 328-341.

[5] Steffen Smolka, Praveen Kumar, Nate Foster, Dexter Kozen, and Alexandra Silva. 2017. Cantor Meets Scott: Semantic Foundations for Probabilistic Networks. In ACM SIGPLAN-SIGACT Symposium on Principles of Programming Languages (POPL), Paris, France. 557-571. 